\title{
Application of Neural Network in hydropower projects
}

\author{
Sixing Zhang ${ }^{1, \text { a }}$ \\ ${ }^{1}$ School of economics and management, North China Electric Power University, 689 Huadian Road, \\ North District, Baoding, Hebei Province, China \\ a2371736732@qq.com
}

Keywords: Neural Network; Hydropower projects; Comprehensive evaluation system; Dimensionless and normalized; Index membership

\begin{abstract}
Hydropower is a clean, renewable and non-polluted energy which can be massive and continuing developed at this moment. Hydropower will help improve of resources and benefits of economy and society. That't to say, the hydropower has the prosperous future and will be kept as high development speed. But hydropower project is characterized as huge investment, long construction period, wide field involvement, complex technology and so on. The purpose of this paper is to research how to ensure the safety and profitability of investments in hydropower projects. According to the case of Xiluodu Hydropower Station, we establish a comprehensive evaluation system, using the Neural Network method. It's proved to be feasible for Neural Network in hydropower projects.Conclusions are put forward finally.
\end{abstract}

\section{Introduction}

China has the most abundant hydropower resource in the world. Hydropower is the sole energy resource which can be massive and continuing developed at this moment. At present, thermal power still accounts for a large proportion in China. However, due to a large amount of coal combustion, waste emissions have seriously polluted the air. Gradually, thermal power industry is showing down. To some extent, water conservancy and hydropower industry will occupy an important position in China's electric power energy projects for a long time. So hydropower is encouraged to be developed by Chinese government to meet with the electricity requirement from the development of economic and society, optimize the allocation of resource and electricity source structure, especially reduce the environment pollution and realize the sustainable development.

In addition, China's energy planning has pointed that the proportion of coal will be reduced to $65 \%$ in primary energy consumption by 2015 . Therefore, the hydropower project appears to be very important. Hydropower investment is becoming increasingly attractive so that power companies have begun to raise funds for the expansion project and some private companies have also optimistic. However, with the progress of China's electricity reform, hydropower investment projects are facing more complex market environment. With the rapid development of the water power industry, how to prepare the feasibility study and the economical evaluation is the key to guarantee the scientific investment decision, improve the investment benefit.

\section{Overview of the comprehensive evaluation of hydropower projects}

Hydropower projects Hydropower project is characterized as huge investment, long construction 
period, wide field involvement, complex technology and so on. In the new competitive market environment, hydropower investment projects will face more uncertainty: First, the price is of uncertainty. Owing to implement of the "bidding", price is not fixed, but fluctuate with supply and demand. Second, the cost of electricity is also changing, which includes bank lending rates, national macroeconomic policies and environmental requirements. Finally, the macroeconomic development and adjustment of industrial structure will bring some risks.

Comprehensive evaluation system Therefore, it's necessary for investment in hydropower projects to assess risks comprehensively to ensure the safety and profitability of investments. Therefore, the comprehensive evaluation of hydropower projects is a multi-factor, multi-target and multi-index problems essentially. The most common methods are: expert advice, analytic hierarchy process, cost-benefit analysis and the Delphi method. But in practice, there are many shortcomings of the methods above. First, the workload is relatively large, especially when the number of samples or survey rounds is large. Second, the evaluation are often subjective.Third, it's not easy to select the appropriate experts, who need to consume a lot of time and effort. Hydropower Investment is often effected by many uncertain factors, so the conventional methods are often difficult to obtain satisfactory results. This paper will use the neural network methods creatively to evaluate hydropower projects , due to its perception, decision-making and control of intelligent behavior.

\section{Neural Network}

Overview Neural networks are an emerging marginal interdisciplinary. Artificial neural networks are relative to the biological neural networks, which is put forward to describe the structure of a biological neural network with a certain simple mathematical model. Using certain algorithms, it can be simulated intelligent behavior of biological neural networks to solve the problems which can not be handled by traditional algorithms. The most widely used is the BP neural network. Artificial neural network is to simulate human thinking. Artificial neural network is a system which is capable of learning, develop the knowledge as well as exceed the level of the original designer. BP neural network is a multi-layer neural network, which was proposed by Rumelhant and Mcclelland in 1986. BP network typically includes one input layer, one or more hidden layer and one output layer. It has a strong nonlinear mapping ability. Its performance advantage mainly reflects in pattern recognition, graphics processing, financial research and function approximation. Thus we use the method to do further forecast below.

Sample training When the network receives a learning mode, the activate value of neurons will be transmitted from input layer neurons through hidden layer neurons to output layer neurons, then the correction connection power will be transmitted from the output layer through the hidden layer, and then back to the input layer level on the basis of the principle of reducing error between the expected output and the actual output. The interval transmission to and fro will go on until the error meets the practical needs. Sample training includes six steps:

step1: Construct initial weight. In order to make the learning process convergence, we initialize the weight randomly with the value varying from -1 to 1 .

step2: Input parameters transmit from the input layer through the hidden layer to output layer.

step3: The output error reversely transmits into input layer.

step4: Cycle interval training between forward back propagation calculation process.

step5: Judge whether a global error meets the requirements.

step6: When the global error is less than the error limit, the network ends the training. Otherwise the 
process turns back to step1, and continues to train.

\section{Specific process}

Indicators established principles It is the basic premise to establish the comprehensive evaluation index system scientifically. Therefore,before selecting indexes, it's necessary to put forward the principles in the evaluation index system. In order to reduce the variables, we transform multiple indexes into several comprehensive indexes based on the following principles.

(1) Hierarchy It refers to the level of the index system should be clearly, which is divided into the goal level, criterion level, index level and program level. The ownership of various indicators should be not duplicate or missing, but clear and definite.

(2) Independence It requires the selected indexes should not be correlative and double-counting problem does not exist between the indicators.

(3) Scientificity It demands the system should have a complete, comprehensive and scientific theoretical basis, which can accurately reflect all aspects of an investment project.

(4) Operability It need consider the complexity, reliability and cost to obtain the indicators, while ensuring that data can be quantified calculations.

Based on the above principles and literature, we have established three index systems: efficiency indexes, cost indexes and qualitative indexes.

Index system For a comprehensive evaluation, it is the first to establish the corresponding evaluation index system. A comprehensive evaluation system includes goal layer, criterion layer, index layer and program layer. Goal layer is achieved by criterion level. Indicators that will affect investment decisions are divided into efficiency indexes, cost indexes and qualitative indexes, which compose the criterion level. Various indexes of index layers are approaches to achieve the total goal, and each program is ultimate measures to achieve the total goal of the system, which can establish a comprehensive evaluation system of hydropower projects.

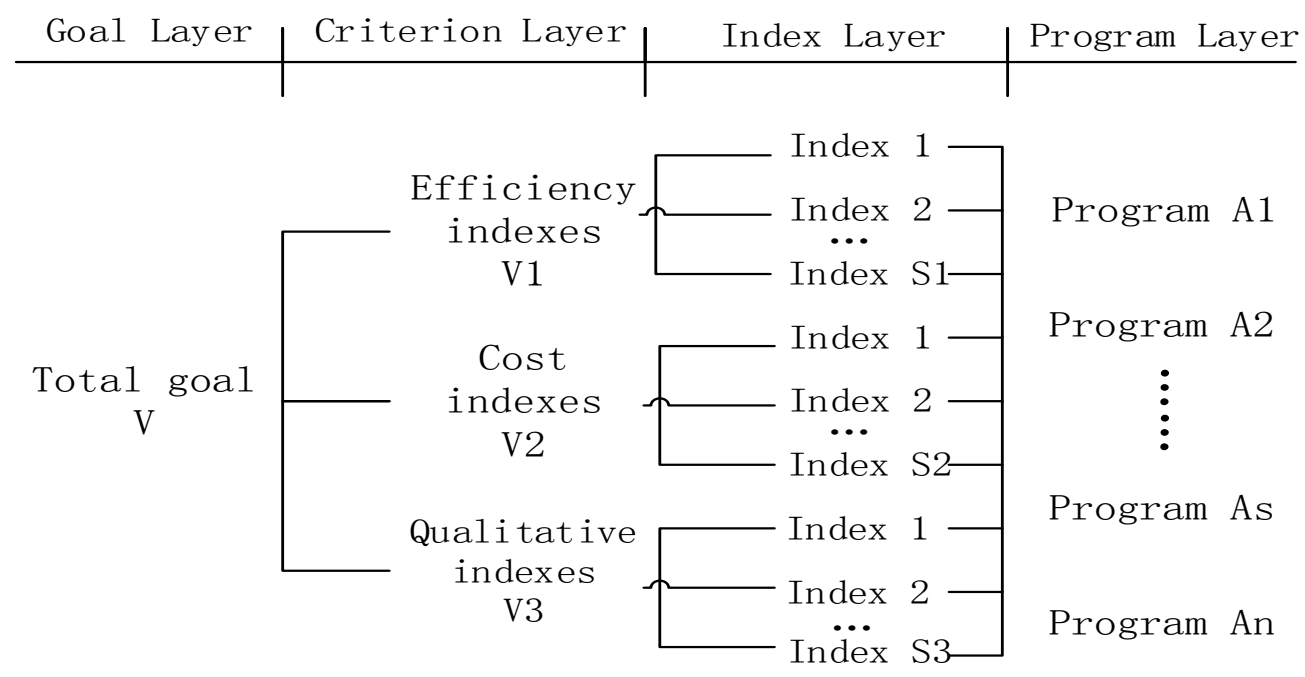

Figure. 1 The index system structure

However, the indexes usually represent different technical and economic parameters. And the dimensions of data are not the same, as far as to a wide difference. So it is necessary to deal with the original data to make them dimensionless and normalized. Treatment methods are as follows:

1) The bigger the value is, the better the efficiency indicator is. Its maximum and minimum values 
respectively are: $\max x_{i j}$ and $\min x_{i j}$, which can be written as follows: $r_{i j}=\frac{x_{i j}-\min x_{i j}}{\max x_{i j}-\min x_{i j}}$

2) The smaller the value is, the better the cost indicator is. Its maximum and minimum values respectively are: $\max x_{i j}$ and $\min x_{i j}$, which can be written as follows: $r_{i j}=\frac{\max x_{i j}-x_{i j}}{\max x_{i j}-\min x_{i j}}$

3) Some evaluation indexes of hydropower projects are qualitative indicators, so it's difficult to obtain quantitative values, such as social benefit, political influence and eco-environmental influence. Decision-makers can only give qualitative indicators and make a judgment.

Application of fuzzy BP neural network. Based on the nature and characteristics of the hydropower industry, we consider the technical and economic level and establish 11 quantitative indicators and 3 qualitative indicators. By collecting authoritative data, we select 10 indexes among them as the sample for training. We normalize first to obtain the relative membership of each index as shown below. The indexes are listed below.

Table 1 Index meaning

\begin{tabular}{ll}
\hline R1: the installed capacity & R2: the guaranteed output \\
\hline R3: the average annual production capacity & R4: the total reservoir storage \\
R5: the annual utilization hours & R6: the internal rate of return \\
R7:the aggregate investment & R8: the total discounted cost \\
R9: the total population migration & R10: the submerged farmland \\
R11: the pay back period & $\begin{array}{l}\text { R12: the degree of driving the development of } \\
\text { local economy }\end{array}$ \\
R13: the degree of impact on the environment & R14: the engineering difficulty
\end{tabular}

Table 2 Membership degree of each index

\begin{tabular}{|c|c|c|c|c|c|c|c|c|c|c|c|}
\hline \multicolumn{2}{|c|}{ Membership } & \multicolumn{10}{|c|}{ Sample number } \\
\hline \multirow{4}{*}{$\begin{array}{l}\text { Efficiency } \\
\text { indicator }\end{array}$} & & 1 & 2 & 3 & 4 & 5 & 6 & 7 & 8 & 9 & 10 \\
\hline & R1 & 0.024 & 0.39 & 0 & 0.451 & 1 & 0.329 & 0.234 & 0.573 & 0.878 & 0.659 \\
\hline & R2 & 0 & 0.438 & 0.15 & 0.658 & 1 & 0.356 & 0.143 & 0.809 & 0.626 & 0.538 \\
\hline & R3 & 0.114 & 0.445 & 0.056 & 0.626 & 1 & 0.348 & 0 & 0.725 & 0.734 & 0.61 \\
\hline \multirow{8}{*}{$\begin{array}{c}\text { Cost } \\
\text { indicator }\end{array}$} & R4 & 0.765 & 0.666 & 0.333 & 0.888 & 0 & 0.555 & 0.344 & 1 & 0.2 & 0.1 \\
\hline & R5 & 1 & 0.848 & 0.969 & 0.901 & 0.34 & 0.787 & 0.674 & 0.992 & 0.219 & 0 \\
\hline & R6 & 1 & 0.826 & 0.982 & 0.801 & 0.102 & 0.781 & 0.672 & 0.844 & 0.1 & 0 \\
\hline & R7 & 0.431 & 0.853 & 0.091 & 0.873 & 1 & 0.7 & 0 & 0.995 & 0.66 & 0.518 \\
\hline & R8 & 0.653 & 0.803 & 0.3 & 0.854 & 0 & 0.658 & 0.166 & 1 & 0.285 & 0.204 \\
\hline & R9 & 0.324 & 0.19 & 0.5 & 1 & 0.541 & 0.324 & 0.923 & 0.735 & 0.887 & 0.596 \\
\hline & R10 & 0.51 & 0 & 0.586 & 0.834 & 1 & 0.413 & 0.536 & 0.908 & 0.266 & 0.835 \\
\hline & R11 & 0.141 & 0.544 & 0.165 & 0.266 & 1 & 0.438 & 0.572 & 0 & 0.837 & 0.51 \\
\hline \multirow{3}{*}{$\begin{array}{l}\text { Qualitative } \\
\text { indicators }\end{array}$} & R12 & 0.25 & 0.5 & 0 & 0.75 & 0.25 & 1 & 0 & 0.25 & 0.5 & 0.75 \\
\hline & R13 & 0.25 & 0.75 & 0.75 & 0.25 & 0.5 & 0.25 & 0.5 & 0 & 1 & 0.75 \\
\hline & R14 & 0.25 & 0.5 & 0.75 & 1 & 0.5 & 0 & 0.75 & 0.5 & 1 & 0.75 \\
\hline \multicolumn{2}{|c|}{ Expected value $\mathrm{E}$} & 0.316 & 0.729 & 0.115 & 0.883 & 0.632 & 0.541 & 0.066 & 0.971 & 0.467 & 0.253 \\
\hline
\end{tabular}


Selection of hidden layer nodes has no theoretical method to guide, so it can be written based experience as follows: $n_{1}=\sqrt{n+m}+a$

Where ${ }^{n}$ : the optimal nodes of hidden layer

$n:$ the nodes of input layer

$m$ :the nodes of output layer

$a$ : a number from 0 to 10

The input index of network is 14 , the output is 1 , so $n_{1}=5$

According to experience, we initialize the learning efficiency of each network layer and determine the values as follow:

the learning efficiency of output layer : $a=0.95$

the learning efficiency of hidden layer: $b=0.85$

error of network requirements 0.0001

In accordance with learning steps of fuzzy neural network, we start to train the model in terms of the sample index membership, the initial value of the weights, the initial value of the learning rate, and error of network requirements. After learning, the error convergence results are as follows:

Table 3 Learning result

\begin{tabular}{lllllllllll}
\hline Sample number & 1 & 2 & 3 & 4 & 5 & 6 & 7 & 8 & 9 & 10 \\
\hline Training number & 0.314 & 0.731 & 0.116 & 0.882 & 0.632 & 0.54 & 0.068 & 0.97 & 0.468 & 0.254 \\
Expected value & 0.316 & 0.729 & 0.115 & 0.883 & 0.632 & 0.55 & 0.066 & 0.971 & 0.467 & 0.253 \\
Absolute error & 0.002 & 0.002 & 0.001 & 0.001 & 0 & 0.01 & 0.002 & 0.001 & 0.001 & 0.001 \\
\hline
\end{tabular}

The learning result

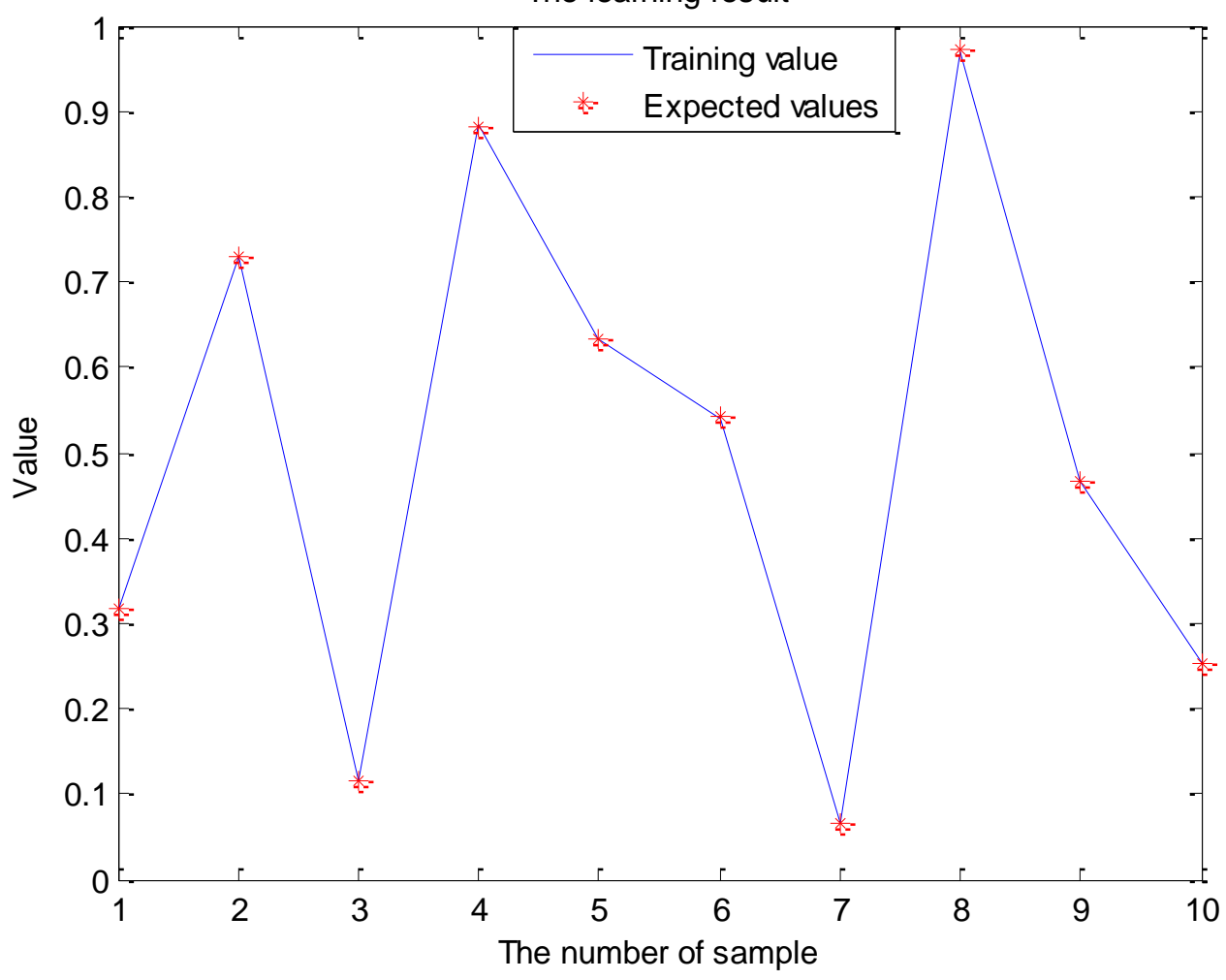

Figure. 2 The curve of error 
Then we can carry out a comprehensive evaluation of alternatives, using fuzzy BP neural network that has already been trained. In this paper, we take Xiluodu hydropower station for example, and technical and economic indicators of the programs are as follows.

Table 4 Values of each index in four programs

\begin{tabular}{lllllllllllllll}
\hline & R1 & R2 & R3 & R4 & R5 & R6 & R7 & R8 & R9 & R10 & R11 & R12 & R13 & R14 \\
\hline & GW & GW & KWH & $\mathrm{m}^{3}$ & Hour & $/$ & Billion & Billion & Person & Mu & Year & $/$ & $/$ & $/$ \\
1 & 1386 & 665.7 & $5712 * 10^{8}$ & $1267 * 10^{8}$ & 4780 & $13.12 \%$ & 792.3 & 1456 & 61035 & 41843 & 17.6 & 0.8 & 0.5 & 0.4 \\
2 & 1312 & 640 & $5650 * 10^{8}$ & $1190 * 10^{8}$ & 4755 & $11.43 \%$ & 757 & 1400 & 61508 & 42583 & 15 & 0.5 & 0.8 & 0.7 \\
3 & 1255 & 654 & $5630 * 10^{8}$ & $1200 * 10^{8}$ & 4600 & $12 \%$ & 800 & 1500 & 63215 & 42121 & 18 & 0.7 & 0.6 & 0.5 \\
4 & 1150 & 661 & $5690 * 10^{8}$ & $1250 * 10^{8}$ & 4715 & $13.01 \%$ & 778 & 1487 & 62125 & 41543 & 14 & 0.6 & 0.5 & 0.6 \\
\hline
\end{tabular}

We carry out non-dimensional treatment to evaluate the programs, combining qualitative indicators, then we get each index membership.

Table 5 Index membership of each membership

\begin{tabular}{lllllllllllllll}
\hline & R1 & R2 & R3 & R4 & R5 & R6 & R7 & R8 & R9 & R10 & R11 & R12 & R13 & R14 \\
\hline 1 & 1.000 & 1.000 & 1.000 & 1.000 & 1.000 & 1.000 & 0.178 & 0.440 & 1.000 & 0.712 & 0.500 & 1.000 & 1.000 & 1.000 \\
2 & 0.686 & 0.000 & 0.244 & 0.000 & 0.861 & 0.000 & 1.000 & 1.000 & 0.783 & 0.000 & 1.000 & 0.000 & 0.000 & 0.000 \\
3 & 0.445 & 0.554 & 0.000 & 0.130 & 0.000 & 0.337 & 0.000 & 0.000 & 0.000 & 0.444 & 0.167 & 0.667 & 0.667 & 0.667 \\
4 & 0.000 & 0.817 & 0.732 & 0.779 & 0.639 & 0.935 & 0.512 & 0.130 & 0.500 & 1.000 & 0.000 & 0.333 & 1.000 & 0.333 \\
\hline
\end{tabular}

We input the index membership degree to the network model and get the evaluation results when it is equal to minimum error.

Table 6 The evaluation results and rankings

\begin{tabular}{ccc}
\hline Programs & Evaluation results & Rankings \\
\hline 1 & 0.86 & 1 \\
2 & 0.24 & 4 \\
3 & 0.71 & 2 \\
4 & 0.45 & 3 \\
\hline
\end{tabular}

\section{Conclusions}

It can be tapped many aspects, using neural network to research and evaluate hydroelectric projects. This research is still limited, the proposed method in this paper needs to be improved. Overall, we think we still have to do further research.

- This paper discusses the hydropower projects evaluation methods, and takes an example to prove its feasibility to apply the fuzzy optimization BP neural network into hydropower investment projects.

- This paper emphasizes to study the application of neural networks in hydroelectric projects. As a matter of fact, it can be combined with some other comprehensive preferred evaluation method. Thus, we can make use of advantages of various methods, making the evaluation results more objective.

- In this paper, we focus on the economic and technical indicators, without considering other qualitative indicators. At present, we can also try to take price and load factor into account.

Since neural network recovered from the early 1980s again, it has become the hot spot and 
developed very rapidly. Besides, it has promptly expanded to many areas in the application and gain fruitful research results. In recent years, many research results of the neural network are mainly in these application areas: pattern recognition, signal management, automatic control, combinatorial optimization, knowledge engineering, management decisions, economic forecasting. In this paper, we only propose a valuable research direction, applying the neural network into the hydropower projects. Its in-depth study will produce more research results.

\section{References}

[1] Information on http://www.mwr.gov.cn/

[2] Information on https://books.google.com.hk/books?id=cMLLBQAAQBAJ\&pg=PA377\&lpg=PA377\&dq=bp+neu ral+network+steps\&source=bl\&ots=xGGsZ73Gw1\&sig=D-tEDJd6gMftcyyiZYTyqDqekx $8 \&$ hl=z $\mathrm{h}-\mathrm{CN} \& \mathrm{sa}=\mathrm{X} \& \mathrm{ei}=1 \mathrm{YLVVMW}$ LqPTmgXthoG4DQ\&ved=0CDwQ6AEwBQ\#v=onepage\&q=bp\% 20neural\%20network\%20steps\&f=false

[3] Changyi $\mathrm{Xu}$, Research on hydropower project coordination development and comprehensive evaluation [D], Tianjin, Tianjin University, 2006

[4] Liying Yin, Research on risk evaluation and index system in hydropower investment project [D], Xi'an, Xian University of Science and Technology

[5] Bing Li, The Research and APPlication of Neural Networks in Power Investment, Hunan University.

[6] Xiongfei Gong, Research on Economic Evaluation in Shaoyang Hydropower Investment Projects, Xiangtan University

[7] Bangzhu Yang, Zhenyu Guo, Introduction to Chinese water conservancy [M], Zhengzhou

[8] Information on http://www.nea.gov.cn/

[9] Zhengchang Zhuo, Big leap - The Road of Sichuan hydropower development, Sichuan hydropower, 2011(03)

[10] Xiaoyu Yuan, Application in selection of reservoir normal water level with multilevel fuzzy optimization method, [D] . Najing, Haihe University 